Revista Brasileira de Agricultura Irrigada v.7, no. 4, p. 235 - 247, 2013

ISSN 1982-7679 (On-line)

Fortaleza, CE, INOVAGRI - http://www.inovagri.org.br

DOI: $10.7127 /$ rbai.v7n400168

Protocolo 168.13 - 02/07/2013 Aprovado em 20/08/2013

\title{
PERDAS DE ÁGUA POR EVAPORAÇ̃̃o E ARRASTE PELO VENTO, EM SISTEMAS DE ASPERSÃO FIXA, NORTE DO ESPÍRITO SANTO
}

\author{
Giselle Sabadim Saraiva ${ }^{1}$, Robson Bonomo ${ }^{2}$, Joabe Martins de Souza ${ }^{3}$
}

\begin{abstract}
RESUMO
O objetivo deste trabalho foi determinar as perdas de água por evaporação e arraste pelo vento por aspersores, de pequeno porte, empregados na irrigação por aspersão na cidade de São Mateus. E comparar os valores obtidos por meio dos testes de campo com os modelos empíricos descrito na literatura. Foram avaliadas as perdas por evaporação e arraste pelo vento para um modelo de aspersor de pequeno porte, bocais de $3,5 \mathrm{~mm}$ x $2,5 \mathrm{~mm}$, operando em quatro pressões, 200, 250, 300 e $350 \mathrm{kPa}$, e em três horários diários, às 05:00, 13:00, e 21:00 horas. Os valores obtidos de perdas de água por evaporação e arraste pelo vento indicaram perdas inferiores a 4\%, e quando foram comparados com aqueles obtidos por modelos de simulação de perdas por evaporação e arraste pelo vento, constatou-se que os modelos empíricos testados não apresentaram boas estimativas em relação aos valores medidos. À uniformidade de aplicação de água, apresentou valores de coeficiente de uniformidade de Christiansen acima de $80 \%$ para as condições de operação e climáticas avaliadas.
\end{abstract}

Palavras-Chave: Eficiência de irrigação, Uniformidade de aplicação de água, Aspersor de pequeno porte.

\section{EVAPORATIVE WATER LOSS AND WIND DRIFT IN FIXED SPRINKLER SYSTEMS, NORTH OF THE ESPÍRITO SANTO}

\begin{abstract}
\footnotetext{
${ }^{1}$ Engenheira Agrônoma, Bolsista da Fapes, Acadêmica do Programa de Pós-Graduação em Agricultura Tropical, Universidade Federal do Espírito Santo, Centro Universitário Norte do Espírito Santo, Rodovia BR 101 Norte, Km. 60, Bairro Litorâneo, 29932-540, São Mateus, ES. E-mail: gizellesab@ hotmail.com

${ }^{2}$ Engenheiro Agrônomo, Doutor em Engenharia Agrícola/Irrigação e Drenagem, Professor da Universidade Federal do Espírito Santo, Centro Universitário Norte do Espírito Santo, Departamento de Ciências Agrárias e Biológicas, Rodovia BR 101 Norte, Km. 60, Bairro Litorâneo, 29932-540, São Mateus, ES. E-mail: robsonbonomo@ceunes.ufes.br

${ }^{3}$ Engenheiro Agrônomo, Mestre em Agricultura Tropical, Doutorando em Produção Vegetal, Universidade Federal do Espírito Santo, Centro de Ciências Agrárias, Programa de Pós Graduação em Produção Vegetal, Alto Universitário s/n, 29500-000, Alegre, ES. E-mail: joabenv@gmail.com
} 


\section{PERDAS DE ÁGUA POR EVAPORAÇÃO E ARRASTE PELO VENTO, EM SISTEMAS DE ASPERSÃO FIXA, NORTE DO ESPÍRITO SANTO}

The objective of this work was to determine the loss of water by evaporation and wind drift for small sprinklers, used in sprinkler irrigation in São Mateus. And compare the values obtained by means of field tests with the empirical models described in literature. We assessed the losses by evaporation and the wind drift to a model of small sprinkler, nozzles of $3.5 \mathrm{~mm} \times 2.5 \mathrm{~mm}$, operating at four pressures, 200, 250, 300 and $350 \mathrm{kPa}$, and three times daily, at 05:00, 13:00 and 21:00 hours. The values of water loss through evaporation and wind drift showed losses of less than $4 \%$, and when they were compared with those obtained by simulation models of evaporation and wind drift losses, it was found that the empirical models tested showed no good estimates of the values measured. For uniformity of water application was reported to Christiansen uniformity coefficient above $80 \%$ for operating and climatic conditions evaluated.

Keywords: Irrigation efficiency, Uniformity of water application, Small sprinklers.

\section{INTRODUÇÃO}

Dentre os sistemas de irrigação comumente empregados na região Norte Capixaba, em especial na cafeicultura, destaca-se os sistemas por gotejamento, e aspersão, na forma de pivô central, aspersão convencional e aspersão fixa. Embora os sistemas por gotejamento apresentem maiores potenciais de uniformidade e eficiência de aplicação de água, há uma grande resistência pelos irrigantes em razão do sistema apresentar problemas de entupimento de emissores em razão da baixa qualidade da água dos mananciais da região, em geral, com elevados níveis de ferro e matéria orgânica em suspensão. Com isso, nos últimos anos tem ocorrido uma tendência na implantação de novos sistemas de aspersão fixa onde se trabalha, em geral, com aspersores de baixa vazão $\left(0,5\right.$ a $\left.1,0 \mathrm{~m}^{3} \mathrm{~h}^{-1}\right)$ e média pressão (200 a $300 \mathrm{kPa})$, com consequente baixa intensidade de aplicação e maiores tempos de irrigação por posição (MANTOVANI et al., 2009).

De acordo com Keller e Bliensner (1990), muita preocupação tem sido relatada à avaliação de sistemas de irrigação, porém perdas de água também reduzem a eficiência do sistema e, frequentemente, os projetistas assumem que os sistemas serão perfeitamente manejados, e as perdas serão mínimas, mas isso nem sempre acontece.
Dentre as perdas merecem destaque as perdas por evaporação e arraste pelo vento, sendo que segundo Christiansen e Davis (1974) e Paz (1990) estas perdas são influenciadas por elementos climáticos como velocidade do vento, umidade relativa, temperatura do ar e radiação solar. A perda de água por evaporação é definida como uma parcela de água que evapora das gotas pulverizadas através do ar, e a perda por arraste, aquela ocorrida quando o vento carrega as gotas para fora da área irrigada (SMAJSTRLA; ZAZUETA, 2003).

$O$ vento tem grande influencia na uniformidade de distribuição, e juntamente com os outros fatores climáticos, aumentam ainda mais as perdas de água por evaporação e arraste pelo vento (MARTIN et al., 2007), sendo que para que ocorra a diminuição destas perdas recomenda-se realizar a irrigação em horários mais apropriados onde a interferência do vento não afete muito a irrigação, diminuindo as perdas.

Além dos elementos meteorológicos, existem outros fatores que afetam estas perdas de água, como a distribuição do tamanho das gotas, que por sua vez é função do diâmetro e da pressão de serviço do bocal, e da distância do percurso da gota no ar. Segundo Playán et al. (2005), reportando trabalho de vários autores, gotas de maiores diâmetros são menos suscetíveis ao arraste pelo vento, uma vez que 


\section{PERDAS DE ÁGUA POR EVAPORAÇÃO E ARRASTE PELO VENTO, EM SISTEMAS DE ASPERSÃO FIXA, NORTE DO ESPÍRITO SANTO}

apresentam menor área de superfície por unidade de massa, e, como consequência, são menos afetadas pelas perdas de água por arraste pelo vento.

As perdas de água por evaporação e arraste pelo vento na irrigação por aspersão podem ser determinadas utilizando-se de métodos de campo, analíticos e de laboratório. Sendo que as perdas no campo variam de 2 a $40 \%$, podendo estar dentro de um intervalo de 10 a $20 \%$, enquanto que os valores de perdas com métodos analíticos e de laboratório apresentam valores de 0,5\% e $2 \%$, respectivamente (BESKOW et al., 2008a; BESKOW et al., 2008b; KINCAID, 1996; KINCAID et al., 1996).

Segundo Beskow et al. (2008a) os ensaios de campo para a determinação de perdas de água por evaporação e arraste pelo vento podem ser realizados com um único aspersor funcionando ou com vários aspersores operando simultaneamente. De acordo com o trabalho de Ortega et al. (2000), as perdas por evaporação e arraste

\section{MATERIAL E MÉTODOS}

Com o intuito de determinar as perdas de água por evaporação e arraste pelo vento, foram realizados 12 testes de campo durante os meses de junho e julho de 2011, estação de inverno, na área experimental localizada no Campus do Centro Universitário Norte do Espírito Santo (CEUNES-UFES), em São Mateus, ES, com latitude $18^{\circ} 43^{\prime \prime} \mathrm{S}$; longitude $30^{\circ}$ 51 " W; e altitude de 39 metros. A região é de clima tropical com inverno seco, do tipo Aw (Köppen), segundo Pezzopane et al. (2010), com temperatura média anual do ar de $23,8^{\circ} \mathrm{C}$ e precipitação pluvial de 1.212 $\mathrm{mm}$.

Os horários dos ensaios avaliados foram às 05:00, 13:00, e 21:00 horas, visando determinar as perdas de água por evaporação e arraste pelo vento, sob pelo vento em sistemas com um único aspersor funcionando é maior quando comparado a sistemas com linhas laterais de aspersores operando simultaneamente. Isso se deve à formação de um microclima gerado pelos sistemas com linhas laterais de aspersores operando simultaneamente.

No Brasil, vários trabalhos foram realizados a fim de avaliar, de alguma forma as perdas de água por evaporação na irrigação por aspersão para as condições climáticas brasileiras, podendo citar os trabalhos realizados por pesquisadores como Azevedo et al. (1999), Beskow et al. (2008a) e Beskow et al. (2008b).

O objetivo deste trabalho foi determinar a uniformidade de aplicação de água e as perdas de água por evaporação e arraste pelo vento em aspersores de pequeno porte, assim como, comparar os valores de perdas obtidos por meio dos testes de campo com os modelos empíricos descrito na literatura.

diferentes condições climáticas, com o sistema operando com quatro aspersores simultaneamente.

Foi empregado o modelo de aspersor Asperjato Júnior®, com ângulo de inclinação de $23^{\circ}$ e bocais de $3,5 \mathrm{~mm} \times 2,5$ $\mathrm{mm}$, representando o valor médio do diâmetro dos bocais, com vazões entre 0,9 a $1,1 \mathrm{~m}^{3} \mathrm{~h}^{-1}$, característicos de aspersores de baixa vazão empregados em sistemas de irrigação por aspersão fixa implantados no norte do estado do Espírito Santo.

As condições meteorológicas durante o período de realização dos testes (Quadro 1) foi registrada por meio de uma estação meteorológica automática, localizada acerca de $20 \mathrm{~m}$ da área de teste, coletando dados de temperatura do ar, umidade relativa do ar e velocidade do vento a cada 10 minutos. 


\section{PERDAS DE ÁGUA POR EVAPORAÇÃO E ARRASTE PELO VENTO, EM SISTEMAS DE ASPERSÃO FIXA, NORTE DO ESPÍRITO SANTO}

Quadro 1. Condições meteorológicas observadas nos dias de realização dos testes.

\begin{tabular}{ccccccccc}
\hline \multirow{2}{*}{ Data } & \multicolumn{3}{c}{ Temperatura $\left({ }^{\circ} \mathrm{C}\right)$} & \multicolumn{3}{c}{ Umidade $(\%)$} & \multicolumn{3}{c}{$\begin{array}{c}\text { Vento a } 10 \mathrm{~m} \\
(\mathrm{~m} / \mathrm{s})\end{array}$} & Chuva \\
& Média & Máx. & Mín. & Média & Máx. & Mín. & Velocidade & $(\mathrm{mm})$ \\
\hline $28 / 06 / 2011$ & 22,3 & 24,2 & 20,3 & 86 & 96 & 75 & 2,57 & 0,0 \\
$04 / 07 / 2011$ & 22,5 & 26,9 & 18,1 & 79 & 97 & 60 & 2,41 & 0,2 \\
$06 / 07 / 2011$ & 22,0 & 27,7 & 16,2 & 78 & 97 & 59 & 1,77 & 0,2 \\
$08 / 07 / 2011$ & 20,5 & 22,3 & 18,7 & 78 & 89 & 67 & 2,86 & 0,0 \\
$19 / 07 / 2011$ & 22,5 & 26,6 & 18,4 & 76 & 96 & 56 & 1,94 & 0,0 \\
$20 / 07 / 2011$ & 21,5 & 26,6 & 16,3 & 72 & 97 & 46 & 1,71 & 0,2 \\
$21 / 07 / 2011$ & 20,9 & 27,1 & 14,7 & 73 & 98 & 47 & 1,86 & 0,0 \\
\hline
\end{tabular}

Os aspersores foram dispostos no espaçamento de 12 por 12 metros, sendo que para cada horário foram realizados testes utilizando pressões de 200, 250, 300 e $350 \mathrm{kPa}$.

Para a avaliação da uniformidade e eficiência de aplicação de água foi instalado, entre quatro aspersores adjacentes, que operaram simultaneamente, um conjunto de pluviômetros, formando uma malha, seguindo metodologia proposta por Merriam e Keller (1978) (Figura 1). O espaçamento entre coletores adotado foi de 1,5 metros entre si. Com base em informações obtidas entre os

Estação agrometeorológica automática

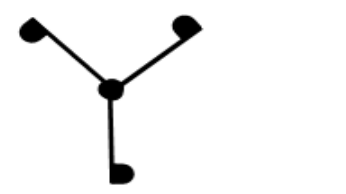

Coletores de referência

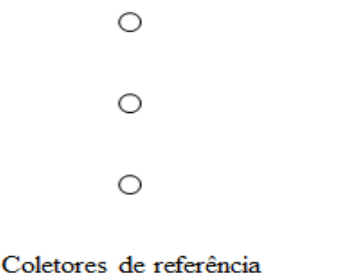

irrigantes da região norte do estado do Espírito Santo, em relação ao tempo de funcionamento do sistema de irrigação, estabeleceu-se que os sistemas ficaram funcionando por um período correspondente à metade do tempo normal de irrigação adotado pelos irrigantes, com duração de 2 horas por teste.

$\mathrm{Na}$ coleta das precipitações foi utilizado, como pluviômetros, coletores de PVC com seção de captação de $50,1 \mathrm{~cm}^{2}$, instalados num suporte com $25 \mathrm{~cm}$ de altura, procurando-se nivelar a seção de captação.

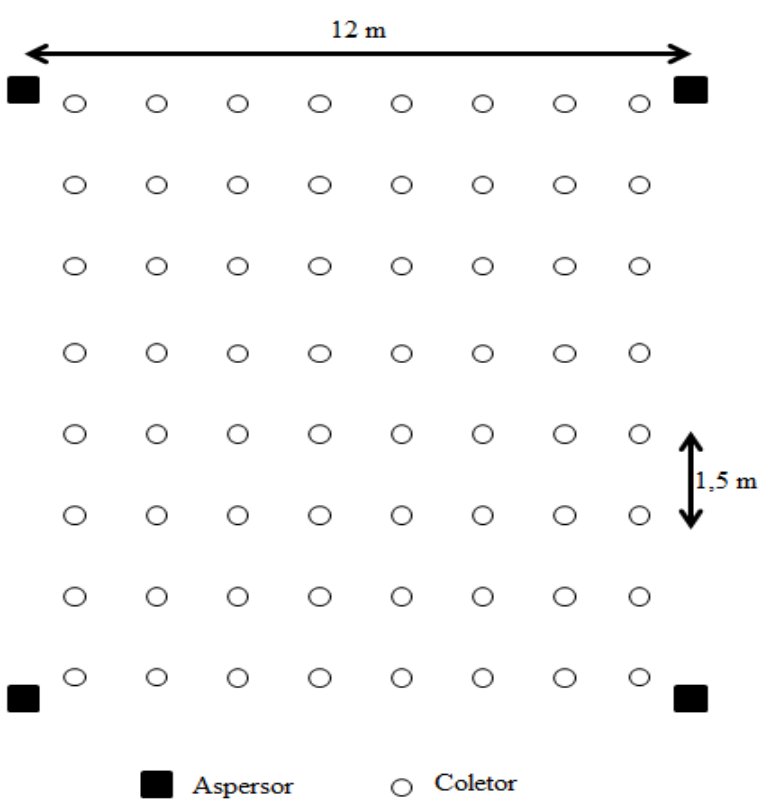

Figura 1. Desenho esquemático da disposição dos coletores, aspersores e estação meteorológica automática, para 0 teste com vários aspersores operando simultaneamente. 


\section{PERDAS DE ÁGUA POR EVAPORAÇÃO E ARRASTE PELO VENTO, EM SISTEMAS DE ASPERSÃO FIXA, NORTE DO ESPÍRITO SANTO}

As medições das pressões na entrada dos aspersores foram realizadas com um manômetro de Bourdon, graduado de 0,0 $6,0 \mathrm{kgf} \mathrm{cm}^{-2}$. A pressão de operação do aspersor foi regulada com uma válvula de gaveta instalada no tubo de subida de cada aspersor. A vazão do aspersor para cada um dos bocais foi medida pelo método direto, onde se mede o tempo necessário para que a água preencha completamente um reservatório com volume conhecido (MERRIAM; KELLER, 1978).

Objetivando estimar a evaporação nos coletores, durante o evento de irrigação e de leitura dos volumes, foi adaptada a metodologia proposta por Tarjuelo et al. (2000) que consiste em dispor cinco coletores como referência, posicionados 15 $\mathrm{m}$ distante da área de testes. Os coletores foram posicionados a $20 \mathrm{~m}$ de distância, para a realização da estimativa de evaporação. Nestes coletores foram colocados volumes correspondentes aos esperados a serem coletados nos testes. Os volumes foram medidos novamente após o término da leitura dos volumes contidos nos coletores. Em cada coletor, o volume de água coletado foi corrigido a partir da evaporação ocorrida durante o teste. Sendo que a leitura de cada coletor foi acrescida com um valor igual à metade da evaporação ocorrida durante o teste, estimada por meio dos coletores de referência.

As perdas de água por evaporação e arraste pelo vento foram determinadas para cada avaliação, pela diferença entre a lâmina média aplicada pelos aspersores dentro da área teste, durante o período de irrigação e a lâmina medida nos coletores. Ressalta-se que as perdas por evaporação ocorridas nos coletores foram ponderadas conforme já descrito. Desta forma, as perdas por evaporação e arraste pelo vento (EA) foram calculadas usando a seguinte equação:

$$
\mathrm{EA}=\frac{\left(L_{\text {maxlic }}\right)-\left(\mathrm{I}_{\mathrm{m} \mathrm{col}}\right)}{\mathrm{I}_{\mathrm{m} \text { aplic }}} 100
$$

em que: $\mathrm{L}_{\mathrm{m}}$ aplic é a lâmina média aplicada, $\mathrm{mm} ; \mathrm{L}_{\mathrm{m} \text { col }}$ é a lâmina média coletada, $\mathrm{mm}$.

As perdas de água por evaporação e arraste pelo vento foram simuladas para as condições dos testes. Para estas simulações foram avaliados, para as condições do momento dos testes, os modelos utilizados por Beskow et al. (2008b), para Lavras, MG, sendo: modelo (A) de Playán et al. (2005), (B) de Tarjuelo et al. (2000), (C) de Trimmer (1987), e (D) de Yazar (1984), além do modelo (E) proposto pelo próprio Beskow et al. (2008b), representados, respectivamente, pelas equações (2), (3), (4), (5) e (6).

$$
\begin{aligned}
& \mathrm{EA}=20,3+0,214 \cdot \mathrm{U}^{2}-2,29 \cdot 10^{-3} \cdot \mathrm{UR}^{2} \\
& \mathrm{EA}=0,007 \cdot \mathrm{P}_{\square}+7,38 \cdot \Delta \mathrm{e}^{0,5}+0,844 \cdot \mathrm{U} \\
& \mathrm{EA}=\left(1,98 \cdot \mathrm{D}^{-0,72}+0,22 \cdot \Delta \mathrm{e}^{0,63}+3,6 \cdot 10^{-4} \cdot \mathrm{P}_{a}^{1,16}+0,14 \cdot \mathrm{U}^{0,7}\right)^{4,2} \\
& \mathrm{EA}=0,003 \exp ^{(0,200)} \cdot(10 \Delta \mathrm{e})^{0,59} \cdot \mathrm{T}^{0,23} \cdot \mathrm{P}_{\mathrm{a}}^{0,76}+0,27 \mathrm{U}^{2,15} \\
& \mathrm{EA}=-0,0177 \cdot \mathrm{P}_{\square}+4,2768 \cdot \Delta \mathrm{e}^{0,5}+3,7499 \cdot \mathrm{U}
\end{aligned}
$$

em que: EA é a perda de água por evaporação e arraste pelo vento, \%; D é o diâmetro do bocal, mm; $\Delta \mathrm{e}$ é o déficit de pressão de vapor, $\mathrm{kPa}$; Ps é a pressão de serviço do aspersor, $\mathrm{kPa}$; $\mathrm{U}$ é a velocidade média do vento, $\mathrm{m} \mathrm{s}^{-1}$; $\mathrm{T}$ é a temperatura do ar, ${ }^{\circ} \mathrm{C}$; e UR é a umidade relativa do ar, $\%$. 


\section{PERDAS DE ÁGUA POR EVAPORAÇÃO E ARRASTE PELO VENTO, EM SISTEMAS DE} ASPERSÃO FIXA, NORTE DO ESPÍRITO SANTO

O déficit de pressão de vapor foi obtido segundo Equação 7 , utilizando-se a Equação 7.1:

$$
\begin{aligned}
& \Delta e=e_{a}(T)-\left(U R \cdot e_{a}(T)\right) \\
& e_{a}=0,61078 \exp \left(\frac{17,269 \cdot t}{t+237_{a}^{3}}\right)
\end{aligned}
$$

em que: e é a pressão de saturação de vapor, em função da temperatura, os demais termos são os mesmos já descritos.

$\mathrm{O}$ indicador estatístico índice de confiança ou desempenho (c) proposto por Camargo e Sentelhas (1996) foi usado para comparar os valores estimados pelos diferentes modelos com os valores observados. O desempenho dos modelos de simulação quanto aos valores de perdas de água por evaporação e arraste pelo vento foram caracterizados da seguinte forma: Ótimo (c > 0,85); Muito Bom (0,76 $\leq \mathrm{c} \geq 0,85)$; $\operatorname{Bom}(0,66 \leq \mathrm{c} \geq 0,75)$; Mediano $(0,61 \leq \mathrm{c} \geq 0,65)$; Sofrível $(0,51 \leq$ $\mathrm{c} \geq 0,60) ; \operatorname{Mau}(0,41 \leq \mathrm{c} \geq 0,50) ; \mathrm{e}$ Péssimo $(\mathrm{c} \geq 0,40)$.

O índice de confiança, "c" (Equação 8), o coeficiente de correlação de Pearson, "r" (Equação 9) e o índice de concordância de Willmott, "d" (Equação 10), segundo trabalho de Silva et al. (2011), foram calculados usando as seguintes equações:

$$
\mathrm{c}=\mathrm{r} \cdot \mathrm{d}
$$

em que: c é o coeficiente de confiança ou desempenho, adimensional; $r$ é o coeficiente de correlação de Pearson; e d é o índice de concordância de Willmott.

$$
\mathrm{r}=\frac{\sum_{\mathrm{i}=1}^{\mathrm{n}}\left(\mathrm{X}_{\mathrm{i}}-\overline{\mathrm{X}}\right) \cdot\left(\mathrm{Y}_{\mathrm{i}}-\overline{\mathrm{Y}}\right)}{\sqrt{\sum_{\mathrm{i}=1}^{\mathrm{n}}\left(\mathrm{X}_{\mathrm{i}}-\mathrm{X}\right)^{2}} \cdot \sqrt{\sum_{\mathrm{i}=1}^{\mathrm{n}}\left(\mathrm{Y}_{\mathrm{i}}-\mathrm{Y}\right)^{2}}}
$$

em que: $r$ é o coeficiente de correlação de Pearson, adimensional; $\mathrm{n}$ é o número de observações; e $X_{i}, \ldots, X_{n}$ e $Y_{i}, \ldots, Y_{n}$ são valores medidos de ambas as variáveis. Os valores médios de $\overline{\mathrm{x}} \mathrm{e} \overline{\mathrm{y}}$ foram calculados, respectivamente, pelas Equações 9.1 e 9.2.

$$
\begin{aligned}
& \overline{\mathrm{x}}=\frac{1}{\mathrm{n}} \cdot \sum_{\mathrm{i}=\mathrm{n}}^{\mathrm{n}} \mathrm{x}_{\mathrm{i}} \\
& \overline{\mathrm{y}}=\frac{1}{\mathrm{n}} \cdot \sum_{\mathrm{i}=\mathrm{n}}^{\mathrm{n}} \mathrm{y}_{\mathrm{i}} \\
& \mathrm{d}=1-\left[\frac{\sum_{\mathrm{i}=\mathrm{i}}^{\mathrm{n}}\left(\mathrm{E}_{\mathrm{i}}-\mathrm{O}_{\mathrm{i}}\right)^{\mathrm{2}}}{\left.\left.\sum_{\mathrm{i}=\mathrm{i}}^{\mathrm{n}}\left[\| \mathrm{E}_{\mathrm{i}}-\sigma\right]+\| \mathrm{O}_{\mathrm{i}}-\sigma\right]\right)^{2}}\right](10)
\end{aligned}
$$

em que: d é o índice de exatidão, adimensional. Os valores de " $d$ " podem variar de 0, para nenhuma concordância, a 1, para uma concordância perfeita; $\mathrm{E}_{\mathrm{i}}$ é o valor estimado; $\mathrm{O}_{\mathrm{i}}$ é o valor observado; é a média dos valores observados; e n é o número de observações.

Para a avaliação da uniformidade de aplicação do sistema foi determinado o Coeficiente de Christiansen (CUC), proposto por Christiansen (1942), para sistemas de aspersão com laterais portáteis. O procedimento consiste na determinação da lâmina de água que atinge o solo, por meio de amostragem feita com coletores distribuídos na superfície irrigada, dispostos em "malha" quadrangular, com espaçamento fixo entre eles. $\mathrm{O}$ volume ou lâmina d'água acumulada em cada coletor, durante $o$ teste, indica a quantidade aplicada na área representada pelo coletor, enquanto a variação entre quantidades coletadas representa o grau de uniformidade da aplicação da água na área.

O coeficiente de uniformidade de Christiansen é representado pela Equação 11, segundo Bernardo et al. (2006).

$$
\mathrm{CUC}=1-\frac{\sum_{\mathrm{i}=\mathrm{i}}^{\mathrm{n}} \| \mathrm{N}_{\mathrm{i}}-\overline{\mathrm{X}} \rrbracket}{\mathrm{nR}}
$$


em que: CUC é o coeficiente de uniformidade de Christiansen, \%; $\mathrm{X}_{\mathrm{i}}$ é a lâmina de água aplicada no i-ésimo ponto

\section{RESULTADOS E DISCUSSÃO}

Os testes de campo realizados em diferentes horários ao longo do dia possibilitou a ocorrência de diferentes condições meteorológicas conforme observado na Tabela 1 , com temperatura sobre a superfície do solo, $\mathrm{mm} ; \overline{\mathrm{X}}$ é a lâmina média de todas as observações, mm; e n é o número de coletores.

do ar variando de 15,80 a $27,25{ }^{\circ} \mathrm{C}$, umidade relativa do ar de 52,34 a $88,48 \%$, velocidade do vento de 0,01 a $2,55 \mathrm{~m} \mathrm{~s}^{-1} \mathrm{e}$ o déficit de pressão de vapor de 0,21 a 1,77 $\mathrm{kPa}$.

Tabela 1. Valores de condições de pressão de serviço $\left(\mathrm{P}_{\mathrm{S}}\right)$, umidade relativa do ar (UR), déficit de pressão de vapor $(\Delta \mathrm{e})$, velocidade do vento $(\mathrm{U})$, temperatura do ar $(\mathrm{T})$, perdas de água por evaporação e arraste pelo vento (EA) e coeficiente de uniformidade de Christiansen (CUC).

\begin{tabular}{ccccccccc}
\hline Data & $\begin{array}{c}\text { Hora } \\
(\mathrm{h})\end{array}$ & $\begin{array}{c}\mathrm{P}_{\mathrm{S}} \\
(\mathrm{kPa})\end{array}$ & $\begin{array}{c}\mathrm{UR} \\
(\%)\end{array}$ & $\begin{array}{c}\Delta \mathrm{e} \\
(\mathrm{kPa})\end{array}$ & $\begin{array}{c}\mathrm{U} \\
\left(\mathrm{m} \mathrm{s}^{-1}\right)\end{array}$ & $\begin{array}{c}\mathrm{T} \\
\left({ }^{\circ} \mathrm{C}\right)\end{array}$ & $\begin{array}{c}\text { EA } \\
(\%)\end{array}$ & $\begin{array}{c}\text { CUC } \\
(\%)\end{array}$ \\
\hline $28 / 06 / 11$ & $5: 00$ & 200 & 76,70 & 0,55 & 2,47 & 20,25 & 3,71 & 81,87 \\
$08 / 07 / 11$ & $13: 00$ & 200 & 65,50 & 0,89 & 2,05 & 21,52 & 2,48 & 81,82 \\
$21 / 07 / 11$ & $21: 00$ & 200 & 85,03 & 0,30 & 0,14 & 17,55 & 0,05 & 86,71 \\
$21 / 07 / 11$ & $5: 00$ & 250 & 77,85 & 0,49 & 0,91 & 19,06 & 0,71 & 87,36 \\
$06 / 07 / 11$ & $13: 00$ & 250 & 59,23 & 1,48 & 1,28 & 27,25 & 0,35 & 85,49 \\
$20 / 07 / 11$ & $21: 00$ & 250 & 79,20 & 0,46 & 0,01 & 19,08 & 0,26 & 86,18 \\
$20 / 07 / 11$ & $5: 00$ & 300 & 88,48 & 0,21 & 0,28 & 15,81 & 2,59 & 87,37 \\
$04 / 07 / 11$ & $13: 00$ & 300 & 52,34 & 1,77 & 1,90 & 26,67 & 1,59 & 88,15 \\
$19 / 07 / 11$ & $21: 00$ & 300 & 85,60 & 0,29 & 0,04 & 17,47 & 0,64 & 84,88 \\
$08 / 07 / 11$ & $5: 00$ & 350 & 77,50 & 0,49 & 2,55 & 18,83 & 0,38 & 81,72 \\
$28 / 06 / 11$ & $13: 00$ & 350 & 55,77 & 1,39 & 2,51 & 24,89 & 1,78 & 80,76 \\
$28 / 06 / 11$ & $21: 00$ & 350 & 86,55 & 0,24 & 0,06 & 15,80 & 1,40 & 83,20 \\
\hline
\end{tabular}

Os valores quantificados, de perdas de água por evaporação e arraste pelo vento (EA), observados na Tabela 1, foram inferiores a $4 \%$, podendo ser considerados baixos, corroborando com os autores Beskow et al. (2008a) e Beskow et al. (2008b), onde encontraram valores de perdas de água por evaporação e arraste pelo vento igual a $4,69 \%$ e valores inferiores a $10 \%$, respectivamente. Estes baixos valores de perdas são devidos aos menores potenciais de evaporação de água no inverno em razão dos baixos valores de temperatura do ar, velocidade do vento e déficit de pressão de vapor.

Em relação ao coeficiente de uniformidade de Christiansen (CUC) pode- 
se verificar na Tabela 1 que os valores obtidos estiveram entre 81,7 a $88,15 \%$ podendo ser considerados, segundo Merriam e Keller (1978), como adequados, considerando CUC adequado quando assume valores acima de $80 \%$. Verifica-se que houve uma tendência de maior uniformidade para as pressões médias de 250 e $300 \mathrm{kPa}$ (Figura 2), bem como para os menores valores de velocidade de vento (Figura 3). Estes valores são similares aos obtidos por Paulino et al. (2009) que trabalhando com aspersão convencional na condições seco, do tipo Aw (Köppen), segundo
Sá Júnior (2009), encontrou valores de CUC de 80,20 \% com pressão de 176,5 $\mathrm{kPa}$. Os mesmo autores trabalhando também com pressão de $210,8 \mathrm{kPa}$ obtiveram um CUC de $74,10 \%$ resultado esse, inferior ao encontrado nesse trabalho. Quanto à velocidade do vento, os autores observaram uma variação de CUC de 82 para a $28 \%$, quando a velocidade do vento aumentou de 1 para $7 \mathrm{~m} \mathrm{~s}^{-1}$, corroborando com os valores encontrados no presente trabalho, onde a variação de CUC passa de 88,15 para $80,76 \%$, quando a velocidade de vento aumenta de 1,90 para 2,51 $\mathrm{m} \mathrm{s}^{-1}$, respectivamente.

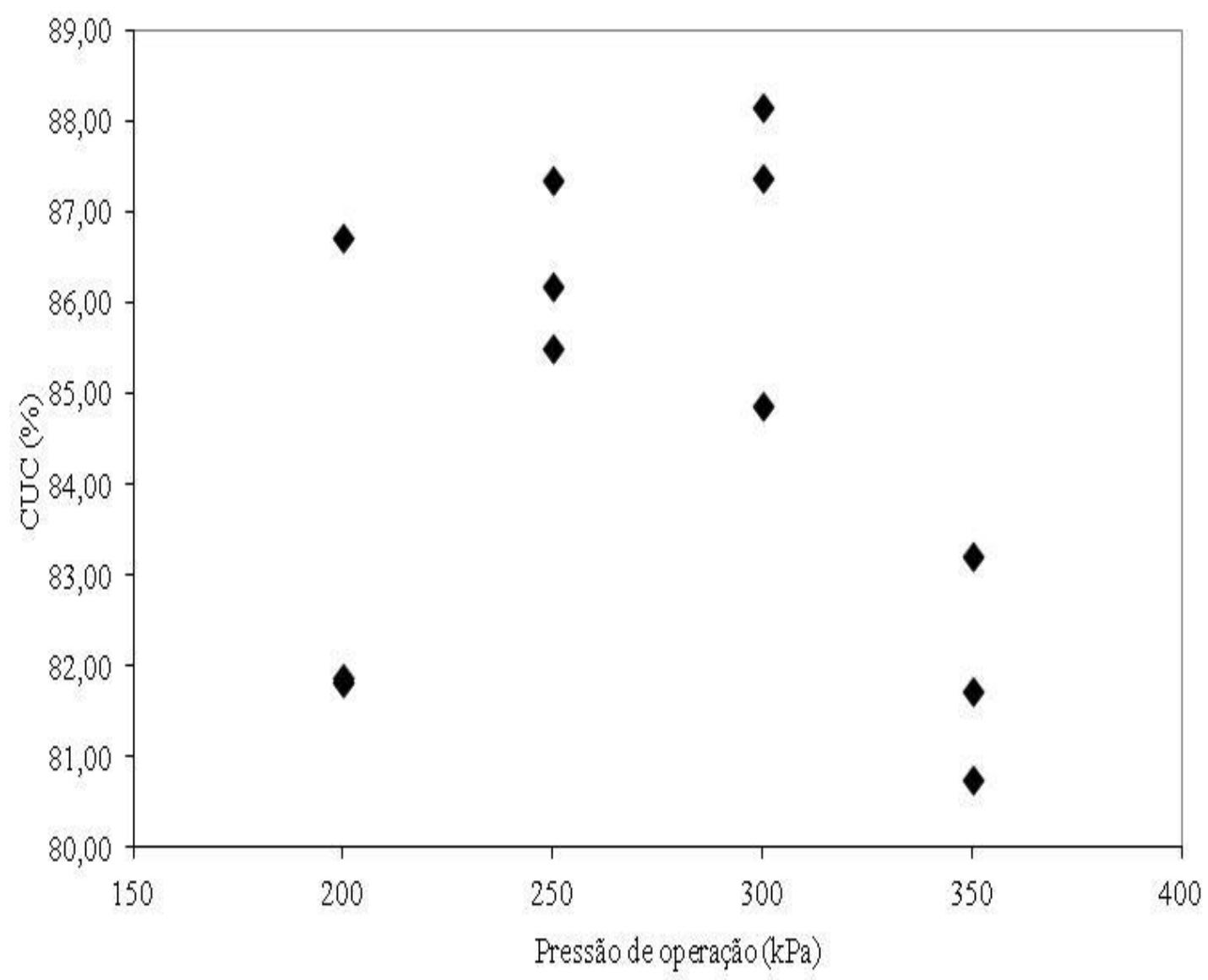

Figura 2. Variação do coeficiente de uniformidade de Christiansen (CUC) em função da pressão de operação dos aspersores. 


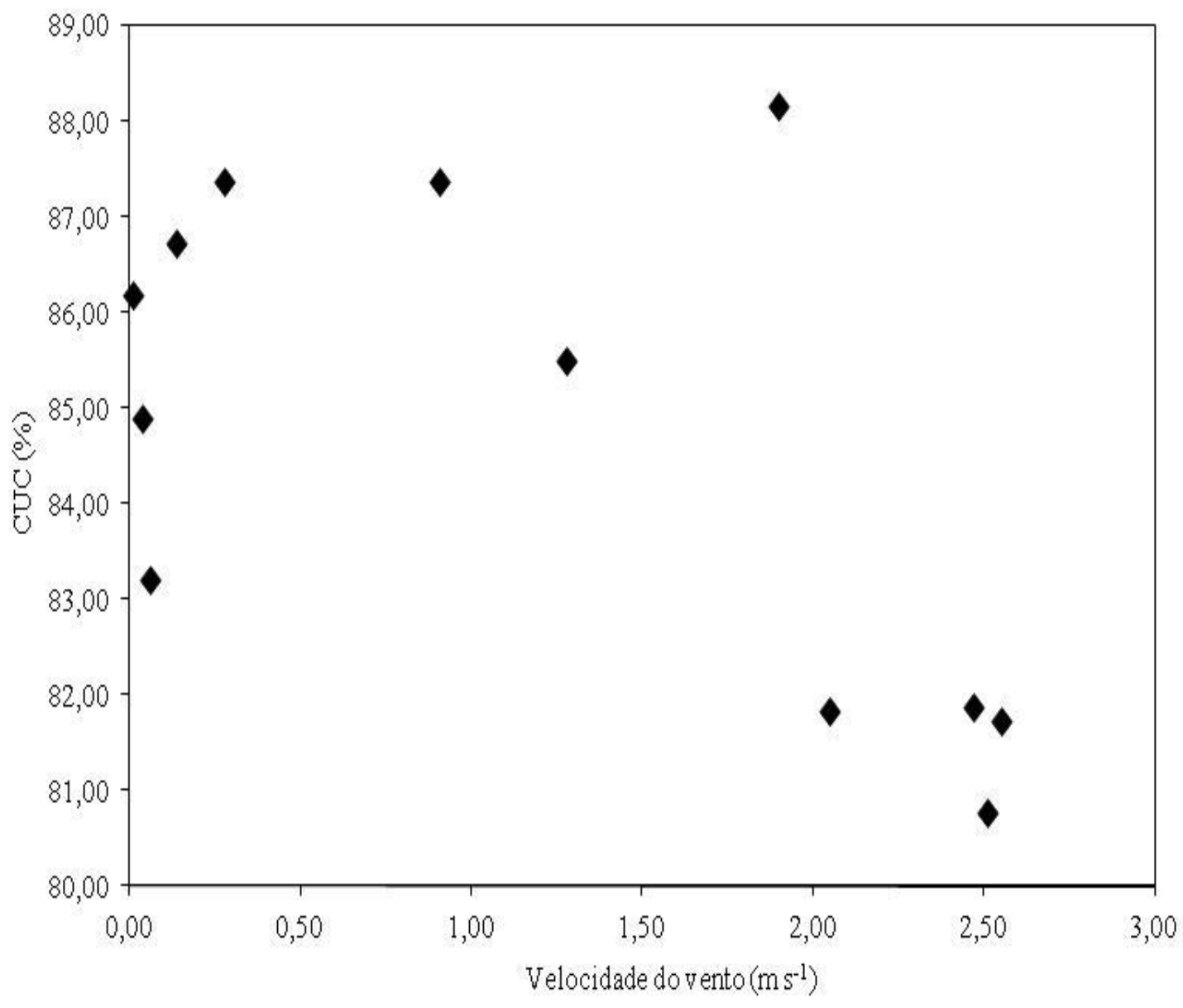

Figura 3. Variação do coeficiente de uniformidade de Christiansen (CUC) em função da velocidade do vento.

Os resultados de uniformidade, em função da pressão de operação e da velocidade do vento, apresentaram maior uniformidade de aplicação de água, e consequentemente, menores perdas de água por evaporação e arraste pelo vento, conforme Figuras 2 e 3, respectivamente. Foram simulados valores de EA para as condições evidenciadas durante os testes, de acordo com os modelos de Playán et al. (2005), Tarjuelo et al. (2000), Trimmer (1987) e Yazar (1984), além do modelo proposto por Beskow et al. (2008b), com geração de gráficos de correlação, expressando a capacidade de cada modelo empírico em prever valores de perdas de água por evaporação e arraste pelo vento.

Os gráficos de correlação apresentam os valores estimados em relação aos valores observados, estando neles indicados também o índice de confiança (c) proposto por Camargo e Sentelhas (1996), o índice de concordância de Willmott (d) e o coeficiente de correlação de Pearson (r), conforme Figura 4. 

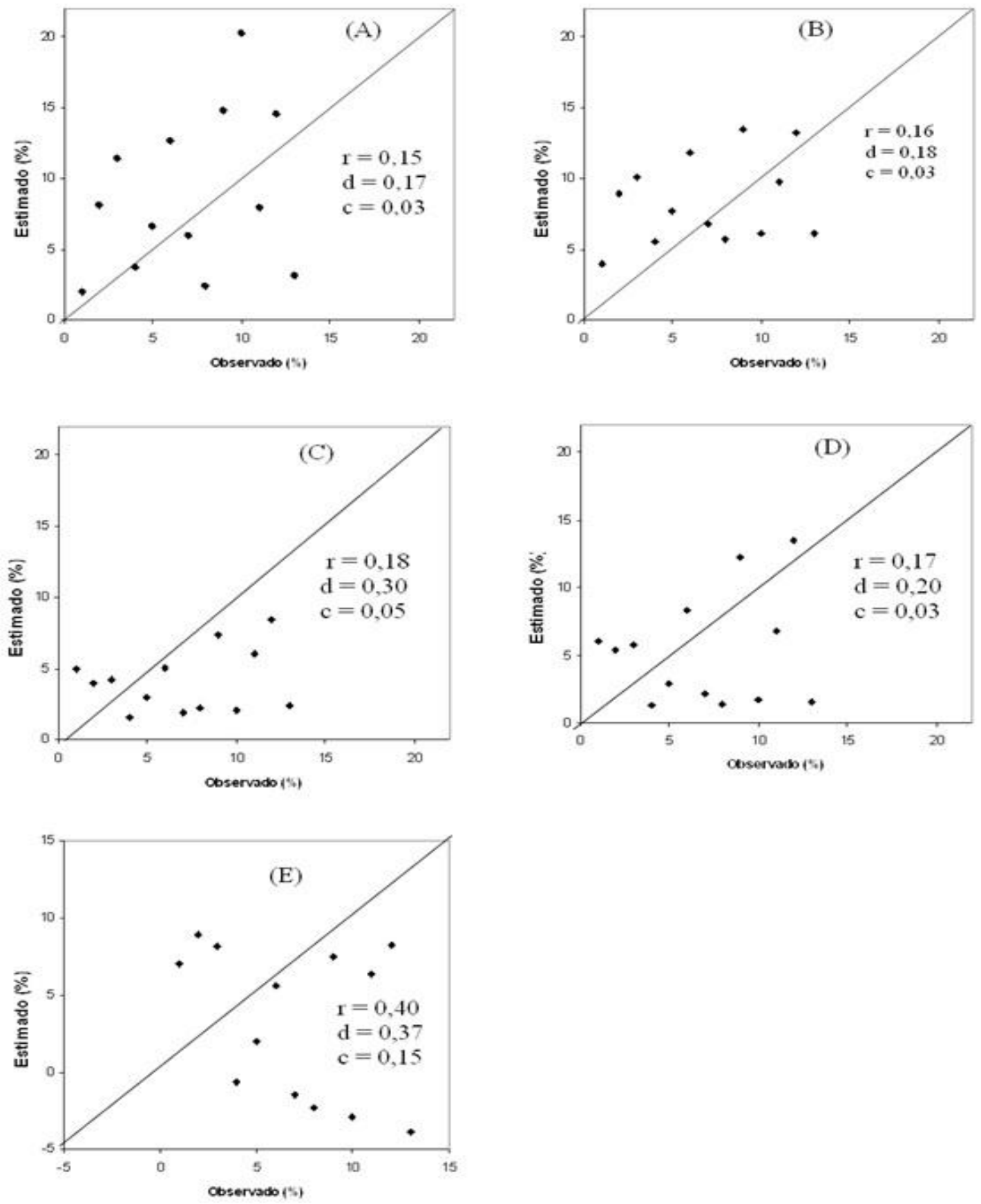

Figura 4. Valores de perdas de água por evaporação e arraste pelo vento observados em testes de campo versus valores simulados pelos modelos: (A) de Playán et al. (2005), (B) de Tarjuelo et al. (2000), (C) de Trimmer (1987), (D) de Yazar (1984), e (E) de Beskow et al. (2008b).

O modelo (A) de Playán et al. (2005), aplicado aos testes da Figura 4A, mostrou desempenho "c" igual a 0,03 (Péssimo), não sendo possível constatar que o modelo apresenta tendência de subestimar ou superestimar os valores de perdas de água por evaporação e arraste pelo vento. Analisando a Figura 4B, é possível constatar que o modelo (B) de Tarjuelo et al. (2000) mostrou desempenho "Péssimo" ("c" igual a 0,03) similarmente ao modelo de Playán. Comportamentos similares também foram observados para os modelos (C) de Trimmer (1987) e (D) de Yazar (1984) nas Figuras 4C e 4D, também 


\section{PERDAS DE ÁGUA POR EVAPORAÇÃO E ARRASTE PELO VENTO, EM SISTEMAS DE ASPERSÃO FIXA, NORTE DO ESPÍRITO SANTO}

apresentando desempenho "péssimo" ("c" igual a 0,03 e 0,05 , respectivamente).

Quando se utilizou o modelo (E) de Beskow et al. (2008b), pôde-se constatar que ele apresentou melhor concordância, apresentados na Figura 4E, mas, mesmo assim, apresentou índice de desempenho classificado como "péssimo" $(\mathrm{c}=0,15)$. O modelo (E) de Beskow et al. (2008b) foi desenvolvido para as condições de Lavras MG, Brasil, aproximando mais das condições operacionais e atmosféricas do presente estudo. Segundo a classificação climática de Köppen, o clima é do tipo Cwa, com inverno seco e verão chuvoso, com precipitação média anual de 1.460

\section{CONCLUSÕES}

As perdas de água por evaporação e arraste pelo vento (EA) foram inferiores a $4 \%$, sendo consideradas baixas;

Os modelos empíricos de estimativa da EA apresentaram desempenho "péssimo", considerando o indicador estatístico índice de confiança ou desempenho aplicado, não sendo possível constatar que os modelos apresentem

\section{REFERÊNCIAS BIBLIOGRÁFICAS}

ALBUQUERQUE, P. E. P.; DURÃES, F. O. M. Uso e manejo de irrigação. Brasília: Embrapa Informação Tecnológica, 2007. 528 p.

AZEVEDO, H. J.; BERNARDO, S.; RAMOS, M. M.; SEDIYAMA, G. C.; CECON, P. R. Influência de elementos do clima no desperdício de energia em um sistema de irrigação por aspersão de alta pressão. Revista Brasileira de Engenharia Agrícola e Ambiental, Campina Grande, v. 3, n. 3, p. 336-341, 1999.

BERNARDO, S.; SOARES, A. A.; MANTOVANI, E. C. Manual de Irrigação. 8. ed. Viçosa: Editora UFV, 2006. 625 p. mm e temperatura média anual de $20,4^{\circ} \mathrm{C}$ (DANTAS et al., 2007).

No entanto, vale salientar que os demais modelos foram obtidos a partir de condições ambientais da Europa e da América do Norte, as quais são distintas das condições ambientais brasileiras, dificultando a importação desse modelo para as condições do Brasil. Por outro lado, mesmo com o desempenho um pouco melhor fica evidente a necessidade de ajuste de um modelo local para a estimativa das perdas de água para as condições locais de irrigação, o que exigirá a realização de um número maior de teste de campo.

tendência de subestimar ou superestimar os valores de EA, sendo que apenas um modelo empírico apresentou melhor concordância com relação às condições ambientais;

O CUC se apresenta acima de $80 \%$, para as condições de pressão de operação do sistema e para as condições de vento avaliadas no estudo.

BESKOW, S.; COLOMBO, A.; PEREIRA, G. M.; TAVEIRA, J. H. S.; RICARDO, C. M. Perdas de água por evaporação e arraste na irrigação por aspersão nas condições climáticas de Lavras-MG, utilizando aspersores de tamanho médio. Revista Irriga, Botucatu, v. 13, n. 1, p. 113-127, 2008a.

BESKOW, S.; COLOMBO, A.; RIBEIRO, M. S.; FERREIRA, L. S.; ROSSI, R. Simulação das perdas de água por evaporação e arraste, no aspersor NY-7 $(4,6 \mathrm{~mm} \times 4,0 \mathrm{~mm})$, em sistemas de aspersão convencional. Revista Engenharia Agrícola, Jaboticabal, v. 28, n. 3, p. 427-437, 2008 b. 
PERDAS DE ÁGUA POR EVAPORAÇÃO E ARRASTE PELO VENTO, EM SISTEMAS DE ASPERSÃO FIXA, NORTE DO ESPÍRITO SANTO

BESKOW, S.; FARIA, L. C.; COLOMBO, A.; MOURA, D. C. M. Modelagem das perdas de água por evaporação e arraste em aspersores de média pressão. Revista Brasileira de Engenharia Agrícola e Ambiental, Campina Grande, v. 15, n. 3, p. 221-228, 2011.

CAMARGO, A. P; SENTELHAS, P. C. Equação para a estimativa da evapotranspiração potencial no estado de São Paulo, baseada no método de Hargreaves - 1974. Revista Brasileira de Agrometeorologia, Santa Maria, v. 4, n. 1, p. 77-81, 1996.

CHRISTIANSEN, J. E. Irrigation by sprinkling. Berkley: University of California, 1942. 124 p.

CHRISTIANSEN, J. E., DAVIS，J. R. Sprinkler irrigation systems. In: HAGAN, R. M., HAISE, H. R., EDMINSTER, T. W. Irrigation of agricultural lands. 3. ed. Madison: American Society of Agronomy, 1974. cap. 11, p. 884-904.

DANTAS, A. A. A.; CARVALHO, L. G.; FERREIRA, E. Classificação e tendências climáticas em Lavras, MG. Revista Ciência e Agrotecnologia, Lavras, v. 31, n. 6, p. 1862-1866, 2007.

KELLER, J.; BLIESNER, R. D. Sprinkle and trickle irrigation. New York: AnaviBook, Van Nostrand Reinhold, 1990. $652 \mathrm{p}$.

KINCAID, D. C. Spraydrop kinetic energy from irrigation sprinklers. Transactions of the American Society of Agricultural Engineers, Saint Joseph, v. 39, n. 3, p. 847-853, 1996.

KINCAID, D. C.; SOLOMON, K. H.; OLIPHANT, J. C. Drop size distributions for irrigation sprinklers. Transactions of the American Society of Agricultural Engineers, Saint Joseph, v. 39, n. 3, p. 839-845, 1996.
KOHL, K. D.; KOHL, R. A.; DeBoer, D. W. Measurement of low pressure sprinkler evaporation loss. Transactions of the American Society of Agricultural Engineers, Saint Joseph, v. 30, n. 4, p. 1071-1074, 1987.

MANTOVANI, E. C.; BONOMO, R.; VICENTE, M. R. Irrigação do café Conilon. In: ZAMBOLIM, L. Tecnologias para produção do café Conilon. Viçosa: Editora UFV, 2009. cap. 10, p. 295-335.

MARTIN, D. L.; KINCAID. D. C.; LYLE, W. M. Design and operation of sprinkler systems. In: HOFFMAN, G. J.; EVANS, R. G.; JENSEN, M. E.; MARTIN, D. L.; ELLIOTT, R. L. Design and operation of farm irrigation systems. 2. ed. Saint Joseph: American Society of Agricultural and Biological Engineers, 2007. cap. 16, p. 557-631.

MERRIAM, J. L., KELLER, J. Farm irrigation system evaluation: a guide for management. Logan: Utah State University, 1978. $271 \mathrm{p}$.

ORTEGA, J. F.; TARJUELO, J. M.; MONTERO, J.; JUAN, J. A. Discharge efficiency in sprinkling irrigation: analysis of the evaporation and drift losses in semiarid areas. Agricultural Engineering International: CIGR Journal, Spain, v. 2, p. 1-21, 2000.

PAULINO, M. A. O.; FIGUEIREDO, F. P.; FERNANDES, R. C.; MAIA, J. T. L. S.; GUILHERME, D. O.; BARBOSA, F. S. Avaliação da uniformidade e eficiência da aplicação de água em sistemas de irrigação por aspersão convencional. Revista Brasileira de Agricultura Irrigada, Fortaleza, v. 3, n. 2, p. 48-54, 2009.

PAZ, V. P. S. Perdas de água e uniformidade de distribuição na irrigação por aspersão. 1990. 59 f. Dissertação (Mestrado em Engenharia 
PERDAS DE ÁGUA POR EVAPORAÇÃO E ARRASTE PELO VENTO, EM SISTEMAS DE ASPERSÃO FIXA, NORTE DO ESPÍRITO SANTO

Agrícola) - Universidade Federal de Viçosa, Viçosa.

PEZZOPANE, J. R. M.; MARSETTI, M. M. S.; SOUZA, J. M.; PEZZOPANE, J. E. M. Condições microclimáticas em cultivo de café conilon a pleno sol e arborizado com nogueira macadâmia. Revista Ciência Rural, Santa Maria, v. 40, n. 6, p. 12571263, 2010.

PLAYÁN, E.; SALVADOR, R.; FACI, J. M.; ZAPATA, N.; MARTINEZ-COB, A.; SÁNCHEZ, I. Day and night wind drift and evaporation losses in sprinkler solidsets and moving laterals. Agricultural Water Management, Amsterdam, v. 76, n. 3, p. 139-59, 2005.

SÁ JÚNIOR, A. Aplicação da classificação de Köppen para o zoneamento climático do Estado de Minas Gerais. 2009. 113 f. Dissertação (Mestrado em Engenharia Agrícola) Universidade Federal de Lavras, Lavras.

SILVA, V. J.; CARVALHO, H. P.; DA SILVA, C. R.; CAMARGO, R.; TEODORO, R. E. F. Desempenho de diferentes métodos de estimativa da evapotranspiração de referência diária em Uberlândia, MG. Bioscience Journal, Uberlândia, v. 27, n. 1, p. 95-101, 2011.

SMAJSTRLA, A. G.; ZAZUETA, F. S. Evaporation loss during sprinkler irrigation. Gainesville: Agricultural and Biological Engineering Department, 2003. $8 \mathrm{p}$.

TARJUELO, J. M.; ORTEGA, J. F.; MONTERO, J.; JUAN, J. A. Modeling evaporation and drift losses in irrigation with medium size impact sprinklers under semi-arid conditions. Agricultural Water Management, Spain, v. 43, n. 3, p. 263284, 2000.

TRIMMER, W. L. Sprinkler evaporation loss equation. Journal of Irrigation and Drainage Engineering, New York, v. 113, n. 4, p. 616-20, 1987.

YAZAR, A. Evaporation and drift losses from sprinkler irrigation systems under various operating condition. Agricultural Water Management, Adana, v. 8, n. 4, p. 439-449, 1984. 